\title{
Dynamic changes in E-cadherin gene promoter methylation during metastatic progression in papillary thyroid cancer
}

\author{
KIRK JENSEN $^{1}$, ANEETA PATEL ${ }^{1}$, VICTORIA HOPERIA ${ }^{2}$, ALEXANDER LARIN ${ }^{2}$, \\ ANDREW BAUER $^{1}$ and VASYL VASKO ${ }^{1}$ \\ ${ }^{1}$ Department of Pediatrics, Uniformed Services University of the Health Sciences, Bethesda, MD 20814, USA; \\ ${ }^{2}$ Center for Endocrine Surgery, Kiev 02091, Ukraine
}

Received January 8, 2010; Accepted March 3, 2010

DOI: $10.3892 /$ etm_00000071

\begin{abstract}
Thyroid cancer cells undergo epithelial to mesenchymal transition (EMT) in the invasive front of tumor. To determine whether the mesenchymal feature of invasive cancer cells is maintained in metastatic sites, we examined E-cadherin methylation and E-cadherin expression in 66 papillary thyroid cancer (PTC) samples and in 34 corresponding lymph node metastases (LNM). Relationships between E-cadherin and cell motility were evaluated using thyroid cancer cell lines. Hypermethylation of the E-cadherin gene promoter was detected in $39.3 \%$ of the PTCs, and loss of E-cadherin expression correlated with lymphocytic infiltration, extrathyroidal invasion and the presence of metastases. Comparing primary PTCs to the corresponding LNM, E-cadherin methylation status was identical in $60 \%$ of the cases. The switch in E-cadherin promoter status from unmethylated in PTCs to hypermethylated in LNM was detected in 5\%; and from hypermethylatated in PTCs to unmethylated in LNM in 35\%. Loss of epigenetic silencing in LNM was associated with a gain of E-cadherin expression. Hypermethylation of the E-cadherin gene promoter was detected in thyroid cancer cell lines with mesenchymal-like morphology. Loss of E-cadherin expression in these cells correlated with high migratory ability. Inhibition of RAS/ERK or PI3K/AKT signaling decreased the migratory ability of these cells but did not induce E-cadherin expression. In the cells with epithelial-like morphology, treatments with phorbol-ester or tumor necrosis factor (TNF)- $\alpha$ resulted in translocation of membranous E-cadherin to the cytoplasm and induction of migration. E-cadherin promoter methylation status and E-cadherin expression were not affected by TNF. Demethylating agents induced apoptosis in the mesenchymallike cells but had no effect on E-cadherin expression or on
\end{abstract}

Correspondence to: Dr Vasyl Vasko, Department of Pediatrics, Uniformed Services University of the Health Sciences, 4301 Jones Bridge Road, Bethesda, MD 20814, USA

E-mail: vvasko@usuhs.mil

Key words: thyroid, cancer, E-cadherin, epigenetic, methylation, metastasis migratory ability. Together, dynamic changes in E-cadherin methylation occur during metastatic progression in thyroid cancer. Epigenetic mechanisms and TNF-inducible signaling independently contribute to the regulation of E-cadherin expression and localization. These mechanisms may play a role in the induction of EMT in primary tumors and in the conversion from the mesenchymal to the epithelial phenotype in metastases.

\section{Introduction}

Papillary thyroid cancer (PTC) is characterized by lymphatic spread, and up to $50 \%$ of patients with PTCs have positive lymph node metastases (LNM) at the time of surgery (1). In addition, lymph nodes are the most common site of thyroid cancer recurrences, and the prevalence of nodal involvement developing after surgery was reported to be 38.5-58.8\%. Therefore, understanding the molecular mechanisms that control thyroid cancer cell invasion and survival in metastatic sites is critical for the development of effective therapy.

Cancer cell invasion and the development of metastases are associated with the induction of epithelial to mesenchymal transition (EMT) in experimental tumors and advanced stage human carcinomas $(2,3)$. Epithelial cells undergoing EMT are characterized by the loss of cell-to-cell contacts and acquisition of a migratory phenotype. The $C D H 1$ (16q22.1) gene encodes the transmembrane glycoprotein E-cadherin, which plays an important role in maintaining cell-cell adhesion in epithelial tissues. Diminished E-cadherin expression has been considered a hallmark of EMT (4).

Activation of signaling pathways due to oncogene mutations contributes to the induction of EMT. Previous studies have shown that HGF/cMET (5), TGF- $\beta$ /Smurf1 (6) and MAPK/ ERK (7) are involved in the regulation of EMT. Epigenetic mechanisms also control EMT through the regulation of E-cadherin expression. Diminished expression of E-cadherin due to its gene promoter hypermethylation has been demonstrated in various malignancies, including leukemia (8), breast cancer (9) and primary colorectal carcinomas (10).

The intracellular location of E-cadherin is critical for its function and is regulated by phosphorylation (11). Activation of tyrosine kinases results in the loss of cadherin-mediated cellcell adhesion by activating E-cadherin endocytosis. The role 
of PKC signaling has been demonstrated in the regulation of E-cadherin trafficking, endocytosis and recycling. It has been shown that cancer cell treatment with phorbol esters increased the rate of E-cadherin endocytosis, resulting in decreased levels of membranous E-cadherin (12). The inflammatory mediator tumor necrosis factor (TNF)- $\alpha$ induces EMT through PKC dependent activation of TGF- $\beta$ (13).

In the thyroid, cDNA microarray analysis and immunohistochemical studies have demonstrated marked down-regulation of E-cadherin expression in poorly differentiated cancer $(14,15)$. Aberrant E-cadherin promoter methylation was demonstrated in $23-83 \%$ of PTCs $(16,17)$.

These studies also suggest that the loss of E-cadherin expression plays a role in the development of thyroid cancer metastases. We recently demonstrated that invasive thyroid cancer cells exhibit a gene expression profile consistent with EMT (18). However, whether mesenchymal features of invasive thyroid cancer cells are maintained in metastatic sites is unknown.

In this study, we examined the methylation status of the E-cadherin gene promoter in a series of PTCs and corresponding LNM. We also used thyroid cancer cell lines to establish relationships between E-cadherin gene expression and cancer cell motility.

\section{Materials and methods}

Thyroid tissue samples. Tumor specimens and clinical data were obtained from an archival tissue bank and the corresponding computerized database, which is maintained under IRB approval of the Walter Reed Army Medical Center. Thyroid tissue samples from 66 patients were used in this study. All tumors were diagnosed as PTCs with H\&E staining according to the WHO classification criteria. Oncogenic mutation status was assessed in all primary PTCs as previously described (18). In 34 cases, samples from LNM were available for analysis.

DNA extraction and methylation-specific PCR. Genomic DNA was prepared from tumor tissues and cell cultures using TRIzol Reagent (Invitrogen, Carlsbad, CA). The sodium bisulfite reaction was carried out on $1 \mu \mathrm{g}$ of DNA according to the protocol specified for the CpGenome ${ }^{\mathrm{TM}}$ DNA Modification kit (Chemicon, Temecula, CA). Methylation-specific PCR (MSP) amplifications of E-cadherin were performed from 2- $\mu 1$ aliquots in a $25-\mu 1$ reaction mixture according to the established protocol for the $\mathrm{CpG}$ WIZ ${ }^{\mathrm{TM}}$ E-cadherin Amplification kit (Chemicon). The amplified products were visualized after electrophoresis on a $2 \%$ polyacrylamide gel. The methylated and unmethylated CDH1 exon $1 \mathrm{CpG}$ islands were 206 and 212 bp, respectively. Genomic DNA from the CpG WIZ ${ }^{\mathrm{TM}}$ E-cadherin Amplification kit was subjected to bisulfite modification and used as a positive control for methylated alleles.

$R N A$ extraction and reverse transcription (RT)-PCR. Total RNA was prepared from tumor tissues and cell cultures using TRIzol Reagent. Using specific primer pairs as previously described, RT-PCR was performed using total RNA, and the products were visualized after electrophoresis on a $2 \%$ agarose gel. cDNA was probed with specific primers for E-cadherin
(CDH1 RT 2 PCR Primer Set; SuperArray Bioscience Corporation, Frederick, MD). The conditions used were as follows: $95^{\circ} \mathrm{C}, 15 \mathrm{~min} ; 40$ cycles of $\left(95^{\circ} \mathrm{C}, 30 \mathrm{sec} ; 55^{\circ} \mathrm{C}, 30 \mathrm{sec}\right.$ and $\left.72^{\circ} \mathrm{C}, 30 \mathrm{sec}\right) 72^{\circ} \mathrm{C}, 10 \mathrm{~min}$. This was confirmed by electrophoresis on a $2 \%$ agarose gel.

Immunohistochemical analysis. Sections were deparaffinized, then soaked in alcohol after microwave treatment in antigen unmasking solution for $10 \mathrm{~min}$. These were then incubated in $3 \%$ hydrogen peroxide for $15 \mathrm{~min}$ to quench endogenous peroxidase activity. Sections were incubated at $4^{\circ} \mathrm{C}$ overnight with anti-E-cadherin antibodies (sc-21791; Santa Cruz Biotechnology, Santa Cruz, CA). Immunostaining was performed by use of the Vectastain Universal Quick kit according to the manufacturer's instructions. Peroxidase staining was revealed in 3,3-diaminobenzidine. A negative control was applied by omission of antiserum. The intensity of staining and cellular localization were evaluated by two independent observers.

For immunofluorescence, species-appropriate secondary antibodies conjugated with Texas Red (Invitrogen) were applied for $1 \mathrm{~h}$ in the dark at room temperature. Cover slides were mounted with the Prolong anti-fade kit (Invitrogen) and examined using fluorescence microscopy.

Cell culture, migration and cell growth. Human thyroid cancer cell lines (TPC1, FTC133, FTC236, FTC238 and WRO82-1) were propagated in RPMI-1640 medium (Invitrogen). Pharmacological inhibitor of PI3K/AKT signaling (LY-294002), TNF- $\alpha$ and 5-aza-2'-deoxycytidine (5-aza) were from Sigma Chemical Co. (St. Louis, MO), and MEK1/2 inhibitor (U-0126) was from Cell Signaling Technology.

Cell migration experiments were performed using a Boyden chamber or Matrigel-coated membrane $(8-\mu \mathrm{m}$ pore size). The efficiency of migration was evaluated by membrane staining using a Diff-Quick staining kit (Dade Behring Inc., Newark, DE). The migration percentage of total cells was calculated as previously described (18). All migration experiments were performed in triplicate.

Cell growth was examined using Vi-CELL ${ }^{\mathrm{TM}}$ Cell Viability Analyzer from Beckman Coulter (Fullerton, CA) according to the manufacturer's instructions.

\section{Results}

Methylation of E-cadherin gene promoter and E-cadherin expression in human thyroid cancer. We examined the methylation status of the E-cadherin promoter by using MSP in PTCs and in thyroid tissue adjacent to the tumor tissue. In PTCs, hypermethylation of the 5' $\mathrm{CpG}$ islands within the E-cadherin promoter was evident in 26/66 (39.3\%) of the cases (Fig. 1A). In the corresponding normal thyroid tissues, methylation of E-cadherin was detected in 1/22 (4.5\%) of the analyzed samples. Morphological evidence of thyroiditis was observed in this case.

Clinicopathological data of the patients as a function of E-cadherin promoter methylation status are presented in Table I. Histological examination of thyroid tumors revealed that the E-cadherin gene promoter was more frequently hypermethylated in PTCs with a typical papillary pattern of growth 
Table I. Clinical features and E-cadherin methylation in papillary thyroid carcinomas.

\begin{tabular}{|c|c|c|c|}
\hline \multirow[t]{2}{*}{ Clinical features } & \multicolumn{2}{|c|}{ E-cadherin promoter methylation status } & \multirow[t]{2}{*}{ P-value } \\
\hline & Hypermethylated (26 cases) & Unmethylated (40 cases) & \\
\hline Age (year) & $43.1 \pm 17.1$ & $35.3 \pm 12.2$ & 0.2949 \\
\hline Tumor size (mm) & $33 \pm 0.6$ & $22 \pm 0.8$ & 0.4902 \\
\hline Gender (male:female) & $6: 20$ & $16: 24$ & 0.1881 \\
\hline Invasion (no:yes) & $4: 22$ & $18: 22$ & 0.0164 \\
\hline Multifocal growth (no:yes) & $12: 14$ & $26: 14$ & 0.2021 \\
\hline Metastases (no:yes) & $1: 25$ & $16: 24$ & 0.0011 \\
\hline
\end{tabular}

compared to the follicular variant of PTCs. Methylation of the E-cadherin promoter was associated with pathological features suggesting an aggressive thyroid cancer such as extrathyroidal invasion and metastases.

Hypermethylation of the E-cadherin promoter was detected in $8 / 30(26.6 \%), 1 / 8(12.5 \%)$ and $0 / 5(0 \%)$ of tumors with BRAF, RET and RAS mutations, respectively. In PTCs without mutations, E-cadherin hypermethylation was detected in $8 / 28(28.5 \%)$ cases. The correlation between E-cadherin promoter methylation and thyroid oncogene mutations was not statistically significant.

E-cadherin expression in the PTCs and normal thyroid tissues was examined by immunohistochemistry. Normal thyroid tissues showed intense membranous staining while the stromal cells did not express E-cadherin (Fig. 1B). Decreased intensity of E-cadherin immunostaining was found in all PTCs with hypermethylated E-cadherin gene promoter, and in 16/40 (40\%) of PTCs with unmethylated E-cadherin. Loss of membranous E-cadherin expression was associated with lymphocyte infiltration of tumor stroma and the presence of metastases. No significant correlation was found between thyroid oncogenic mutation status and the level of E-cadherin protein expression.

Methylation of the E-cadherin promoter in the invasive front of thyroid cancer and in lymph node metastases. We hypothesized that thyroid cancer cells located in the invasive front of thyroid cancer acquire increased motility due to hypermethylation of the E-cadherin promoter and decreased E-cadherin expression. Therefore, we examined samples dissected from the invasive front and central areas of the same tumor in 10 widely invasive PTCs. In 7 cases, the methylation patterns of the E-cadherin promoter and patterns of E-cadherin immunostaining were the same in the central and invasive areas. In 3 cases, there was a switch in E-cadherin promoter methylation status from unmethylated in the central area to hypermethylated in the invasive area of the tumor.

We also compared the methylation status of the E-cadherin promoter between the primary PTC and LNM in 34 cases, for which both primary tumors and corresponding metastases were available for analysis. In 20 of the 34 examined cases (60\%), methylation of the E-cadherin promoter was identical in the primary tumor and in the corresponding LNM (Fig. 1C). The E-cadherin promoter was unmethylated in both the primary tumor and in the corresponding LNM in 10 cases.
In 10 cases, the E-cadherin promoter was hypermethylated in both the primary tumor and in the corresponding LNM. In $14 / 34(40 \%)$ cases, the methylation status of E-cadherin was different in the primary PTC and in the corresponding LNM. In 12 cases (35\%), the E-cadherin gene promoter was hypermethylated in the primary PTC but unmethylated in LNM. Immunostaining showed an increased level of E-cadherin expression in these LNM (Fig. 1D). In two cases (5\%), a hypermethylated E-cadherin gene promoter was detected only in metastases, and not in the primary tumor.

E-cadherin expression and migratory ability of thyroid cancer cell lines. To determine the role of E-cadherin in the regulation of thyroid cancer cell motility, we examined the methylation status of the E-cadherin promoter in thyroid cancer cell lines with different phenotypes. TPC-1, FTC133, FTC236 and FTC238 cells showed spindle, mesenchymal-like morphology, but WRO cells demonstrated round, epitheliallike characteristics. In mesenchymal-like thyroid cancer cells, the E-cadherin promoter was hypermethylated, and E-cadherin expression was not detected at the mRNA and protein levels. In the epithelial-like thyroid cancer cell lines, unmethylated copies of the E-cadherin promoter were detected, and E-cadherin expression was evident at the mRNA and protein levels. Immunostaining demonstrated membranous patterns of E-cadherin expression in epithelial-like WRO cells (Fig. 2A).

E-cadherin-negative thyroid cancer cells with mesenchymal-like morphology efficiently migrated in the Boyden chamber assay. These cells were also able to invade through the Matrigel-coated membrane. By contrast, epithelial-like E-cadherin-positive WRO cells did not migrate (Fig. 2B).

To determine whether E-cadherin methylation status and the migratory ability of thyroid cancer cells is affected by treatment with demethylating agents, we incubated thyroid cancer cells with 5-aza-2'-deoxycytidine. Thyroid cancer cell treatment with 5 -aza (2.5 and $5 \mu \mathrm{M}$ for up to $72 \mathrm{~h}$ ) did not affect the methylation status of the E-cadherin gene promoter or E-cadherin expression, and did not inhibit cell migration. In mesenchymal like-cells, 5-aza treatment was associated with inhibition of cell growth. By contrast, 5-aza treatment had no significant effect on growth in the epithelial-like thyroid cancer cells.

We previously demonstrated that PI3K/AKT and RAS/ ERK signaling plays a role in the regulation of thyroid cancer cell migration. It has also been shown that the activation of 
A

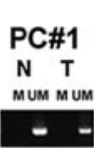

B

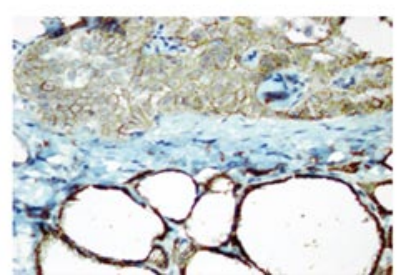

C

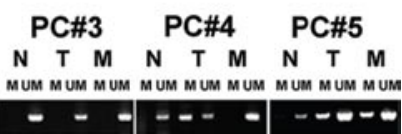

D

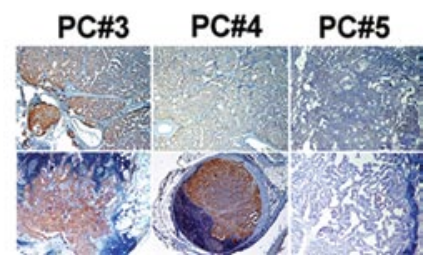

Figure 1. (A) Methylation-specific PCR (MSP) in papillary thyroid cancer PC\#1 is representative of most tumor samples with no change in the methylation status vs. normal tissue. PC\#2 is representative of $39 \%$ of the tumor samples which were methylated. (B) E-cadherin IHC comparing the tumor to normal tissue. Decreased intensity of staining in the tumor (top) compared to the normal thyroid (bottom). (C) MSP in tumor and metastases. Three representative cases. Normal tissue is unmethylated in all cases. PC\#3 demonstrates unmethylated E-cadherin promoter in the tumor and metastasis (M). PC\#4 exhibits marked induction of methylation in the primary tumor, but reversion to an unmethylated state in metastatsis. PC\#5 methylation in the primary tumor and metastasis. (D) E-cadherin IHC comparing the tumor to metastases. E-cadherin expression correlates with methylation status. Gain of E-cadherin expression in metastatic lesions compared to the primary tumor is shown in PC\#4. M, methylated; UM, unmethylated; T, tumor; $\mathrm{N}$, normal tissue.

PKC signaling by PMA induces morphological changes in WRO cells.

To determine whether these signaling control thyroid cancer cell motility through the regulation of E-cadherin, we examined also E-cadherin promoter methylation status and E-cadherin expression after cell exposure to PI3K/AKT inhibitor (LY-294002), RAS/ERK inhibitor (U-0126) and after treatment with PMA.

In mesenchymal-like thyroid cancer cells treatment with LY-294002 $(20 \mu \mathrm{M})$ and $\mathrm{U}-0126(20 \mu \mathrm{M})$ was associated with the inhibition of migration. However, these migratory inhibitory effects were not associated with changes in E-cadherin promoter methylation status, E-cadherin expression and conversion to an epithelial phenotype. PMA treatment had no significant effect on mesenchymal-like thyroid cancer cell migration, but dramatically altered the morphology of epithelial-like cells. PMA treatment (100 $\mathrm{nM}$ for $24 \mathrm{~h})$ was associated with loss of cell-to-cell contacts and acquisition of mesenchymal like morphological features in WRO cells. Immunostaining showed that chronic exposure to PMA was associated with the loss of membranous E-cadherin staining in WRO cells (Fig. 2C) and the induction of migration (Fig. 2D). PMA-inducible re-localization of E-cadherin from the cell membrane to the cytoplasm was not associated with changes in E-cadherin promoter methylation status or in the level of E-cadherin expression.

Since decreased membranous E-cadherin expression in human PTCs was associated with lymphocyte infiltration, we hypothesized that these effects may be mediated by the cytokine TNF- $\alpha$. Chronic TNF- $\alpha$ treatment (100 nM for $24 \mathrm{~h}$ )

A

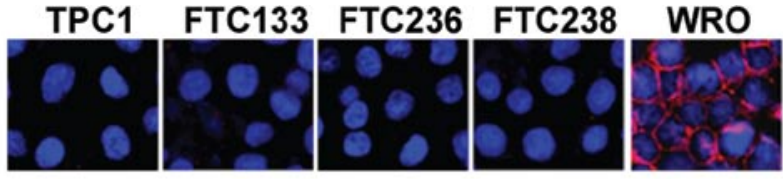

B

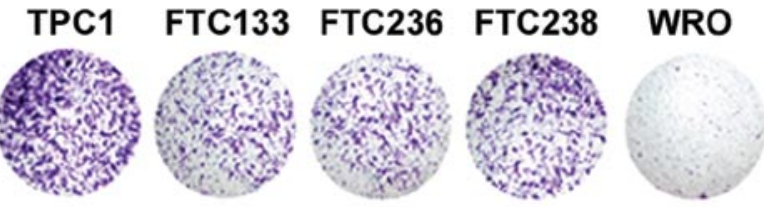

C

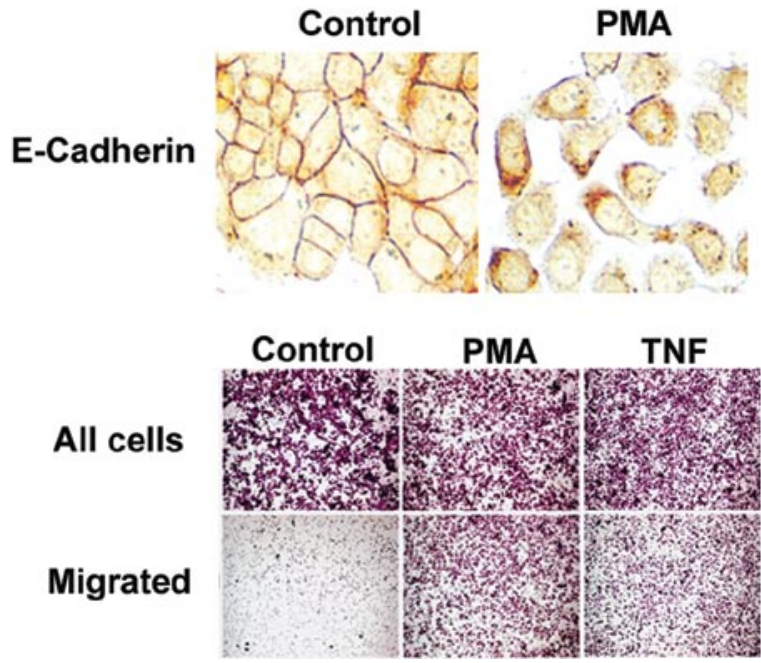

Figure 2. (A) E-cadherin expression in thyroid cancer cell lines. WRO cells were characterized by membranous E-cadherin expression. (B) Migratory ability of thyroid cancer cells. Microphotograph was taken after cells were removed from the upper surface of the membrane. Staining indicates only migrated cells. E-cadherin-negative cells readily migrate, but E-cadherinexpressing WRO cells do not migrate. (C) E-cadherin localization in WRO cells. Treatment with PMA results in the loss of membranous E-cadherin and loss of cell-to-cell contacts. (D) PMA and TNF treatments induce migration in WRO cells.

resulted in the loss of membranous E-cadherin and induction of cancer cell migration (Fig. 2D). Similarly to PMA, TNF-inducible re-localization of E-cadherin from the cell membrane to the cytoplasm was not associated with changes in E-cadherin promoter methylation status or in the level of E-cadherin expression.

To determine whether constitutive TNF stimulation is required for the maintenance of the mesenchymal phenotype in thyroid cancer cells we performed time course experiments. Analysis of thyroid cancer cells showed that the TNF-inducible loss of membranous E-cadherin expression was transient. The switch from medium containing TNF to TNF-free medium resulted in the re-establishment of epithelial like morphology and loss of migratory ability in WRO cells.

\section{Discussion}

The acquisition of a migratory phenotype is an essential property of invading and metastasizing cancer cells. Cell migration is commonly understood as the movement of individual cells that undergo polarized extension-contraction cycles. Reduced 
expression of E-cadherin is regarded as one of the main molecular events involved in individual cancer cell invasion.

In the present study, we demonstrated that PTCs presenting with metastases at the time of surgery more frequently display E-cadherin promoter methylation compared to PTCs without metastases. These findings are consistent with previously reported data showing a high frequency of aberrant E-cadherin promoter methylation in poorly differentiated metastatic thyroid cancers $(19,20)$. Our data suggest that thyroid cancer cells with the highest density of methylation and the most diminished E-cadherin protein expression may be responsible for dissociation from the primary tumor and dissemination to lymph nodes.

Comparative analysis of E-cadherin in primary tumors and corresponding LNM revealed dynamic changes in E-cadherin methylation status and E-cadherin expression during metastatic progression. In a significant proportion of cases, E-cadherin expression was increased in lymph node metastases compared to primary tumors, and this phenomenon was related to the loss of epigenetic inhibition. These results suggest that the mesenchymal phenotype of invading cancer cells may be reversed in metastatic sites, and thyroid cancer cells may undergo a mesenchymal to epithelial transition in lymph node metastases.

The possible role of mesenchymal to epithelial transition in metastatic sites is supported by previously reported data showing that epigenetic inactivation of E-cadherin is unstable and may be influenced by micro-environmental factors during breast cancer metastatic progression (21). There is evidence suggesting that E-cadherin plays a role in cancer cell survival within the lymphatic and metastatic sites. Strong expression of E-cadherin was observed in intra-lymphatic emboli in patients with breast lobular carcinomas (22). The re-expression of E-cadherin was demonstrated in lymph node metastasis from E-cadherin-negative colorectal carcinomas (23).

We found that, in certain cases, PTCs presenting with metastases were characterized by E-cadherin expression in both the primary and metastatic lesions. These findings suggest that thyroid cancer metastases may arise from tumor cells that have never lost E-cadherin expression. Previous studies underlined the role of E-cadherin in the regulation of a specific type of cellular translocation referred to as collective cell migration $(24,25)$. In contrast to single-cell migration, during collective cell migration cancer cells maintain cell-cell contact and migrate as a group of cells forming a protruding sheet or nest. In the course of tumor progression, tumor clusters may enter lymphatic vessels and can be isolated from peripheral blood (26). It is possible that thyroid cancer cells may use this mechanism and invade lymphatic vessels as a group of interconnected cancer cells expressing a high level of cell adhesion molecules.

To explore the possible role of E-cadherin in the regulation of thyroid cancer cell migration, we performed in vitro studies. Consistent with human data, our in vitro results demonstrated a direct link between the methylation-associated loss of E-cadherin expression and thyroid cancer cell motility. Thyroid cancer cells with the highest density of methylation and the most diminished E-cadherin protein expression were characterized by a high migratory ability.
We also explored the relationships between the activation of thyroid oncogene-inducible signaling and epigenetic mechanisms in the regulation of thyroid cancer cell motility. Our results indicate that PI3K/AKT and RAS/ERK signaling contributes to thyroid cancer cell motility independently of E-cadherin methylation, E-cadherin expression and localization. By contrast, the effects of TNF-inducible signaling on cell migration involve changes in intracellular E-cadherin localization.

To determine whether the pharmacological targeting of methylation induces E-cadherin expression and converts mesenchymal-like cells to an epithelial phenotype, we examined the effects of 5-aza on thyroid cancer cells. Though treatment with 5-aza did not induce E-cadherin expression, it was associated with the inhibition of growth in mesenchymallike thyroid cancer cells. Clinical trials in humans have shown the anti-neoplastic activity of 5-aza (Decitabine) in patients with leukemia and myelodysplastic syndrome, and a clinical trial evaluating Decitabine in the treatment of patients with metastatic thyroid cancer is ongoing. Our data showed that E-cadherin-positive and -negative thyroid cancer cells are characterized by different responses to treatment with 5-aza, and suggested that a demethylating agent may have a variable effect against cancer cells located in primary tumors and in metastatic lesions.

In summary, in the present study we demonstrated that E-cadherin expression in thyroid cancer cells is regulated by epigenetic mechanisms, and dynamic changes in E-cadherin methylation status may occur during metastatic progression. Epigenetic mechanisms and activation of TNF-inducible signaling independently contribute to thyroid cancer cell invasiveness through regulation of E-cadherin expression and intracellular E-cadherin localization. These mechanisms may play a role in the induction of epithelial to mesenchymal transition in the primary tumor, as well as in the conversion from the mesenchymal to the epithelial phenotype in lymph node metastases.

\section{Acknowledgements}

This study was funded by the Department of Pediatrics, Uniformed Services University of the Health Sciences (USUHS). We thank Ildy Katona, Chair, Department of Pediatrics, for the support of this project.

\section{References}

1. Puxeddu E and Filetti S: The 2009 American Thyroid Association Guidelines for Management of Thyroid Nodules and Differentiated Thyroid Cancer: progress on the road from consensus- to evidence-based practice. Thyroid 19: 1145-1147, 2009.

2. Shook D and Keller R: Mechanisms, mechanics and function of epithelial-mesenchymal transitions in early development. Mech Dev 120: 1351-1383, 2003.

3. Thiery JP: Epithelial-mesenchymal transitions in development and pathologies. Curr Opin Cell Biol 15: 740-746, 2003.

4. Takeichi M: Cadherin cell adhesion receptors as a morphogenetic regulator. Science 251: 1451-1455, 1991.

5. Matteucci E, Ridolfi E and Desiderio MA: Hepatocyte growth factor differently influences Met-E-cadherin phosphorylation and downstream signaling pathway in two models of breast cells. Cell Mol Life Sci 63: 2016-2026, 2006. 
6. Wang H, Radjendirane V, Wary $\mathrm{KK}$ and Chakrabarty $\mathrm{S}$ : Transforming growth factor beta regulates cell-cell adhesion through extracellular matrix remodeling and activation of focal adhesion kinase in human colon carcinoma Moser cells. Oncogene 23: 5558-5561, 2004.

7. Honma N, Genda T, Matsuda Y, Yamagiwa S, Takamura M, Ichida $\mathrm{T}$ and Aoyagi $\mathrm{Y}$ : MEK/ERK signaling is a critical mediator for integrin-induced cell scattering in highly metastatic hepatocellular carcinoma cells. Lab Invest 86: 687-696, 2006.

8. Corn PG, Smith BD, Ruckdeschel ES, Douglas D, Baylin SB and Herman JG: E-cadherin expression is silenced by 5 ' $\mathrm{CpG}$ island methylation in acute leukemia. Clin Cancer Res 6: 4243-4248, 2000.

9. Gagnon J, Shaker S, Primeau M, Hurtubise A and Momparler RL: Interaction of 5-aza-2'-deoxycytidine and depsipeptide on antineoplastic activity and activation of 14-3-3sigma, E-cadherin and tissue inhibitor of metalloproteinase 3 expression in human breast carcinoma cells. Anticancer Drugs 14: 193-202, 2003.

10. Lind GE, Thorstensen L, Lovig T, Meling GI, Hamelin R, Rognum TO, Esteller M and Lothe RA: A CpG island hypermethylation profile of primary colorectal carcinomas and colon cancer cell lines. Mol Cancer 3: 28, 2004.

11. Jaggi M, Rao PS, Smith DJ, Wheelock MJ, Johnson KR, Hemstreet GP and Balaji KC: E-cadherin phosphorylation by protein kinase D1/protein kinase $\mathrm{C}\{\mathrm{mu}\}$ is associated with altered cellular aggregation and motility in prostate cancer. Cancer Res 65: 483-492, 2005.

12. Le TL, Joseph SR, Yap AS and Stow JL: Protein kinase C regulates endocytosis and recycling of E-cadherin. Am J Physiol Cell Physiol 283: C489-C499, 2002.

13. Takahashi E, Nagano O, Ishimoto T, Yae T, Suzuki Y, Shinoda T, Nakamura S, Niwa S, Ikeda S, Koga $H$, Tanihara $H$ and Saya $H$ : TNF-\{alpha\} regulates TGF-\{beta\}-dependent epithelial-mesenchymal transition by promoting hyaluronan-CD44-Moesin interaction. J Biol Chem 285: 4060-4073, 2010.

14. Fluge O, Bruland O, Akslen LA, Lillehaug JR and Varhaug JE: Gene expression in poorly differentiated papillary thyroid carcinomas. Thyroid 16: 161-175, 2006.

15. Choi YL, Kim MK, Suh JW, Han J, Kim JH, Yang JH and Nam SJ: Immunoexpression of HBME-1, high molecular weight cytokeratin, cytokeratin 19, thyroid transcription factor-1, and E-cadherin in thyroid carcinomas. J Korean Med Sci 20 853-859, 2005

16. Graff JR, Greenberg VE, Herman JG, Westra WH, Boghaert ER, Ain KB, Saji M, Zeiger MA, Zimmer SG and Baylin SB: Distinct patterns of E-cadherin $\mathrm{CpG}$ island methylation in papillary, follicular, Hurthle's cell, and poorly differentiated human thyroid carcinoma. Cancer Res 58: 2063-2066, 1998.
17. Hoque MO, Rosenbaum E, Westra WH, Xing M, Ladenson P, Zeiger MA, Sidransky D and Umbricht CB: Quantitative assessment of promoter methylation profiles in thyroid neoplasms. J Clin Endocrinol Metab 90: 4011-4018, 2005.

18. Vasko V, Espinosa AV, Scouten W, He H, Auer H, Liyanarachchi S, Larin A, Savchenko V, Francis GL, de la Chapelle A, Saji M and Ringel MD: Gene expression and functional evidence of epithelial-to-mesenchymal transition in papillary thyroid carcinoma invasion. Proc Natl Acad Sci USA 104: 2803-2808, 2007.

19. Brecelj E, Frkovic Grazio S, Auersperg $M$ and Bracko $M$ : Prognostic value of E-cadherin expression in thyroid follicular carcinoma. Eur J Surg Oncol 31: 544-548, 2005.

20. Rocha AS, Soares P, Fonseca E, Cameselle-Teijeiro J, Oliveira MC and Sobrinho-Simoes M: E-cadherin loss rather than beta-catenin alterations is a common feature of poorly differentiated thyroid carcinomas. Histopathology 42: 580-587, 2003.

21. Graff JR, Gabrielson E, Fujii H, Baylin SB and Herman JG: Methylation patterns of the E-cadherin 5' $\mathrm{CpG}$ island are unstable and reflect the dynamic, heterogeneous loss of E-cadherin expression during metastatic progression. J Biol Chem 275: 2727-2732, 2000.

22. Gupta A, Deshpande CG and Badve S: Role of E-cadherins in development of lymphatic tumor emboli. Cancer 97: 2341-2347, 2003.

23. Batistatou A, Charalabopoulos AK, Scopa CD, Nakanishi Y, Kappas A, Hirohashi S, Agnantis NJ and Charalabopoulos K: Expression patterns of dysadherin and E-cadherin in lymph node metastases of colorectal carcinoma. Virchows Arch 448: 763-767, 2006.

24. Friedl P, Noble PB, Walton PA, Laird DW, Chauvin PJ, Tabah RJ, Black M and Zanker KS: Migration of coordinated cell clusters in mesenchymal and epithelial cancer explants in vitro. Cancer Res 55: 4557-4560, 1995.

25. Nabeshima K, Moriyama T, Asada Y, Komada N, Inoue T, Kataoka H, Sumiyoshi A and Koono M: Ultrastructural study of TPA-induced cell motility: human well-differentiated rectal adenocarcinoma cells move as coherent sheets via localized modulation of cell-cell adhesion. Clin Exp Metastasis 13: 499-508, 1995.

26. Brandt B, Junker R, Griwatz C, Heidl S, Brinkmann O, Semjonow A, Assmann $\mathrm{G}$ and Zanker KS: Isolation of prostatederived single cells and cell clusters from human peripheral blood. Cancer Res 56: 4556-4561, 1996. 